\title{
Experimental Investigations on Humidity-Sensing Behaviour of Neodymium Oxide
}

\author{
Bal Chandra Yadav*, Richa Srivastava, Monika Singh and Alok Kumar ${ }^{1}$ \\ Nanomaterials and Sensors Research Laboratory \\ Department of Physics, Lucknow University, Lucknow-226007, India \\ 'Department of Mineral Physics, Geological Survey of India, \\ Northern Region, Lucknow, U.P., India
}

(Received August 3, 2008; accepted December 2, 2008)

Key words: humidity sensor, sensitivity, annealing, resistance

In this paper, we report the humidity-sensing behaviour of neodymium oxide (99\% pure, Johnson \& Matthey, London). It was characterized by scanning electron microscopy (SEM), X-ray diffraction (XRD) and by measuring the mass loss. From SEM, the average particle size is $60-90 \mathrm{~nm}$ and particles have a random pore size. XRD reveals that at room temperature the sensing material is $\mathrm{Nd}(\mathrm{OH})_{3}$ and is crystalline in nature. When neodymium oxide was annealed at $200^{\circ} \mathrm{C}$ and at higher temperatures, it became $\mathrm{Nd}_{2} \mathrm{O}_{3}$. The particle size calculated from Scherrer's formula varies from 100 to $110 \mathrm{~nm}$. A pellet of this material was made using a hydraulic pressing machine (M.B. Instruments, New Delhi) for use as a sensing element after thermal annealing at temperatures of $200,400,600$ and $800^{\circ} \mathrm{C}$. After each step of annealing, the sensing element was placed in a specially designed conductivity holder and exposed to humidity inside a chamber with controlled humidity. Variations in resistance due to the adsorption of water vapour through the sensing element were observed. The sensitivity of the sensing element, the repeatability and the effect of temperature on morphology and sensing characteristics were studied.

\section{Introduction}

Humidity plays a very important role in the environment as well as in human life. ${ }^{(1-2)}$ It adsorbs heat and an appropriate level of humidity is necessary for industrial processes. Manufactured products are packed in a low or moisture-free environment. Human discomfort occurs if the humidity level in the environment becomes either too low or too high. The measurement of humidity gives us an estimate of the amount of water vapour present in the air. Some of the reasons for the importance of humidity measurement ${ }^{(3-5)}$ are listed below:

"Corresponding author: e-mail: balchandra_yadav@rediffmail.com 
1. In many production and manufacturing processes, the quality of the end product depends on controlling the humidity of the surrounding environment.

2. Human health, productivity and comfort are adversely affected by extremely humid conditions.

3. Humidity sensing and control are important in the optimal functioning of most home appliances.

4. Because of the discharge of electrostatic energy that accumulates in solid-state electronic equipment in a dry environment, humidity control is necessary to ensure the optimal functioning of instruments.

The increasing importance of measuring humidity has motivated researchers to search for more reliable and cost-effective humidity sensors and related control systems. There have been many approaches considered with the aim of developing a feasible humidity measurement technique.

Rare-earth oxides (REOs) have a tremendous range of applications in optics, solidstate electronics and transparent optoionic devices. They have high resistivity $\left(\rho \sim 10^{15}\right.$ $\Omega \mathrm{cm})$, a high dielectric constant $(\epsilon=7-20)$, a large band gap $(4-6 \mathrm{eV})$ and a high recrystallization temperature. $\mathrm{Nd}_{2} \mathrm{O}_{3}$ is an REO that exhibits a hygroscopic property leading to the formation of hydroxides. This hygroscopic nature affects the electrical properties $^{(6-9)}$ of sensing materials.

\section{Preparation of Sensing Element}

Six hundred milligrams of neodymium oxide ( $99 \%$ pure, Johnson \& Matthey, London) was used, and after vigorous grinding for $6 \mathrm{~h}$, was mixed well with $10 \%$ glass powder. The addition of glass powder as a permanent binder during the process plays a major role in increasing the adhesiveness of the material, enabling the formation of a pellet. The pellet with $3 \mathrm{~mm}$ thickness and $9 \mathrm{~mm}$ diameter was made at a pressure $79 \mathrm{MPa}$ using a hydraulic pressing machine (M.B. Instruments, New Delhi) at room temperature, and it was used as a sensing element. This prepared pellet was thermally annealed at temperatures of $200,400,600$ and $800^{\circ} \mathrm{C}$ to study the effects of temperature on the morphology and sensing characteristics.

\section{Material Characterization}

\subsection{Scanning electron micrographs}

Figure 1 shows a scanning electron micrograph of the pellet at room temperature. Micropores can be seen in the photograph. The size of the particles varies from 60 to 90 $\mathrm{nm}$. Figure 2(a) shows a micrograph of a pellet annealed at $200^{\circ} \mathrm{C}$. Larger and random pores are observed for the pellet annealed at $400^{\circ} \mathrm{C}$, as shown in Figs. 2(b) and 2(c).

\subsection{X-Ray diffraction}

Figure 3(a) shows XRD patterns of the neodymium oxide in powder form at room temperature. It reveals that the material consists of $\mathrm{Nd}(\mathrm{OH})_{3}$ with a small amount of impurity. The XRD pattern of the sensing material annealed at $400^{\circ} \mathrm{C}$ is shown in 


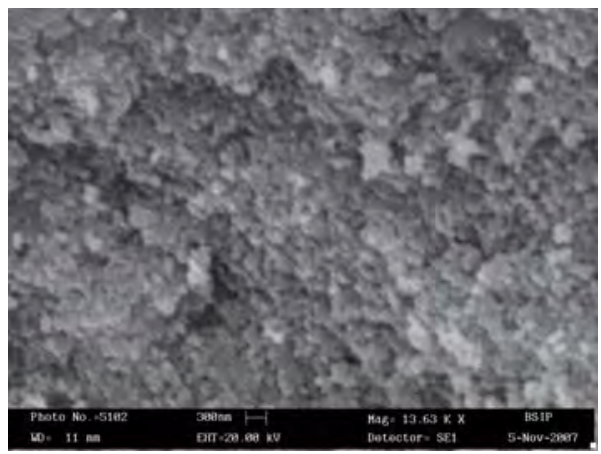

Fig. 1. Scanning electron micrograph of pellet of sensing material at room temperature.

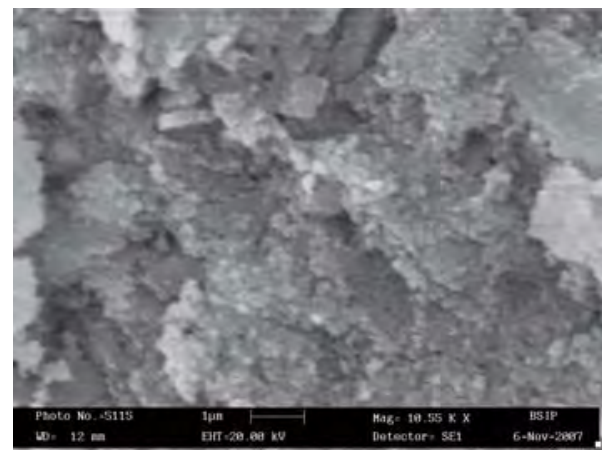

(a)

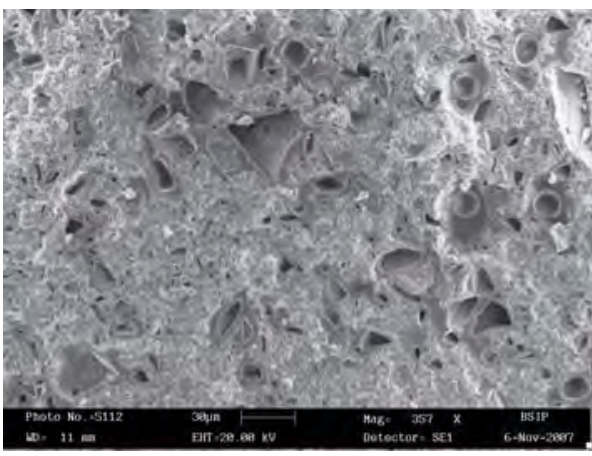

(b)

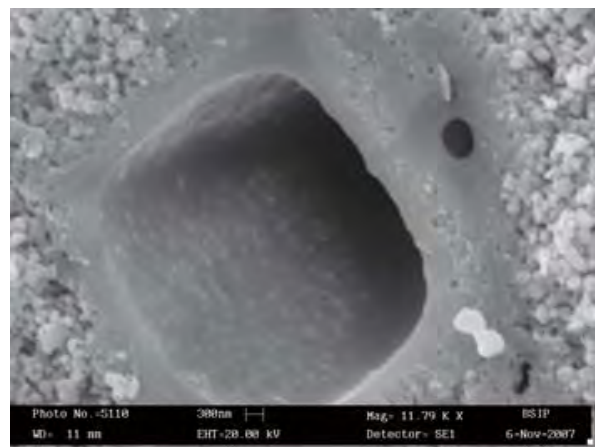

(c)

Fig. 2. (a) Scanning electron micrograph of pellet of sensing material annealed at $200^{\circ} \mathrm{C}$; (b) scanning electron micrograph of pellet of sensing material annealed at $400^{\circ} \mathrm{C}$ at microscale; (c) scanning electron micrograph of pellet of sensing material annealed at $400^{\circ} \mathrm{C}$ at nanoscale. 
(a)

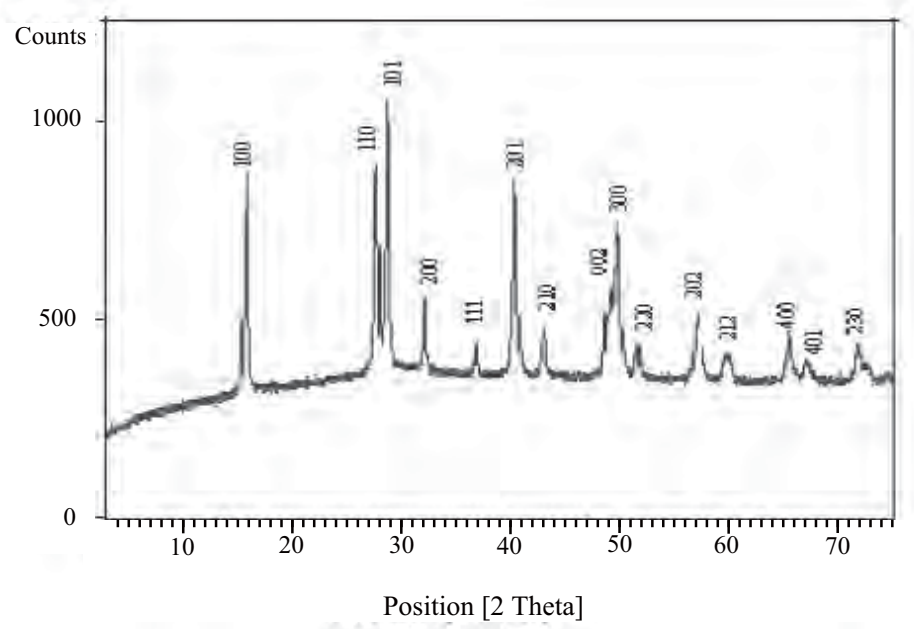

(b)

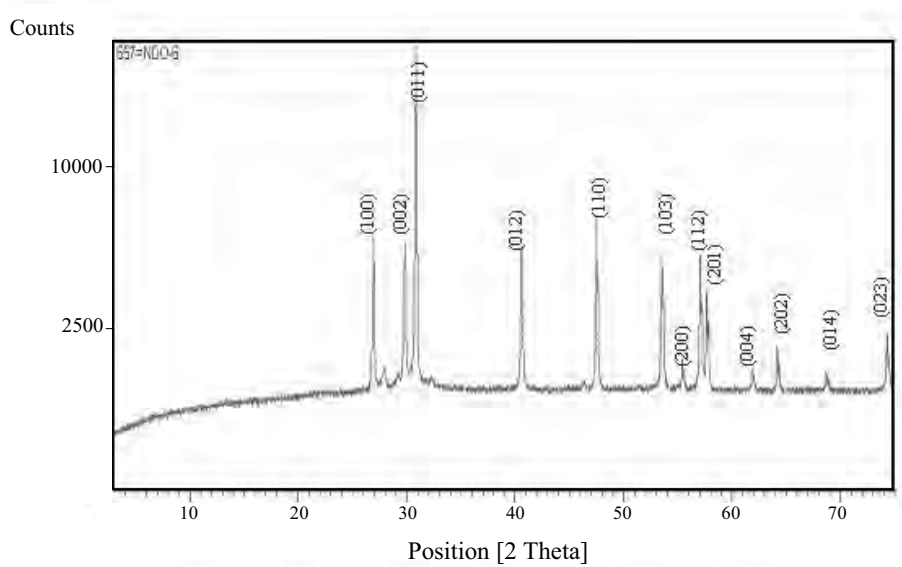

Fig. 3. (a) XRD pattern of $\mathrm{Nd}_{2} \mathrm{O}_{3}$ powder (unannealed); (b) XRD pattern of $\mathrm{Nd}_{2} \mathrm{O}_{3}$ powder annealed at $400^{\circ} \mathrm{C}$.

Fig. 3(b). All the peaks correspond to $\mathrm{Nd}_{2} \mathrm{O}_{3}$ with improved crystallinity. The lattice parameters are $a=3.8310, b=3.8310$ and $c=5.9990$. The crystal system is hexagonal. The average crystallite size $(D)$ of the sensing material can be calculated by the Scherrer's formula, which is given by

$$
D=\frac{K \lambda}{\beta \cos \theta}
$$


where $K=0.94$ is Scherrer's coefficient, which depends on the shape of the crystallite and the type of defects present, $\lambda$ is the wavelength of $\mathrm{X}$-ray radiation, $\beta$ is the full width at half maximum (FWHM) of the diffraction peak and $\theta$ is the angle of diffraction. The particle size calculated from Scherrer's formula varied from 100 to $110 \mathrm{~nm}$.

\subsection{Percentage mass loss}

As the sensing material is hygroscopic, its mass loss was investigated and the percentage variation of consistency in mass of the sensing material after annealing at 200, 400, 600 and $800^{\circ} \mathrm{C}$ for $2 \mathrm{~h}$ was observed. The result is shown in Fig. 4 and shows that as the annealing temperature increases, the mass of the sensing element decreases owing to the removal of water vapour.

\section{Experimental Method}

The experimental setup in this study consists of a controlled humidity chamber, a hygrometer, a thermometer and a digital multimeter. A saturated solution of potassium hydroxide was used as a dehumidifier and a saturated solution of potassium sulphate was used as humidifier. A pellet, which was prepared as a sensing element, was placed in a conductivity-measuring holder with a $\mathrm{Cu}$ electrode-pellet- $\mathrm{Cu}$ electrode arrangement and then was exposed to humidity inside a specially designed controlled humidity chamber. The humidifier/dehumidifier was kept in a dish over a stand. During the experiments, the temperature of the chamber remained constant. In the first step of the experiment, the chamber was dehumidified to $15 \% \mathrm{RH}$ using the dehumidifier and then humidity in the chamber was produced using the humidifier. The accuracy of the hygrometer (Huger, Germany) used here is $1 \% \mathrm{RH}$ and that of the thermometer is $1{ }^{\circ} \mathrm{C}$. Each sensing element was thermally annealed at temperatures of $200,400,600$ and $800^{\circ} \mathrm{C}$ for $3 \mathrm{~h}$ in an electric furnace. After each step of annealing, the pellet was exposed to humidity. Variations in the resistance with changes in relative humidity were recorded.

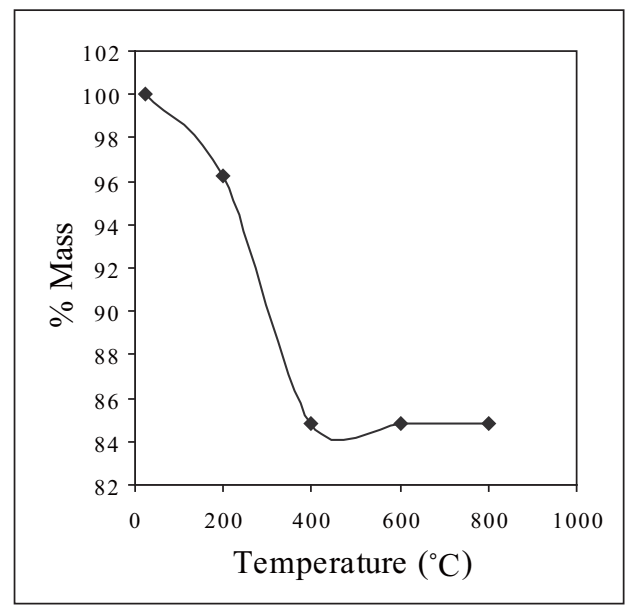

Fig. 4. Percentage of mass of sensing material vs annealing temperature. 


\section{Principle of Sensor Operation}

A ceramic humidity sensor exhibits chemical resistance. The conductivity of a ceramic material varies with the amount of water adsorbed through its surface. ${ }^{(10)}$ This principle is employed for the measurement of moisture in all resistive-type humidity sensors.

The sensitivity of a humidity sensor has been defined as the change in resistance $(\Delta R)$ of the sensing element per unit change in relative humidity $(\% \mathrm{RH}),{ }^{(1)}$ i.e.,

$$
S=\frac{\Delta R}{\Delta \mathrm{RH} \%}(\mathrm{M} \Omega / \% \mathrm{RH}) .
$$

The sensitivity of the sensor after annealing at different temperatures was calculated. The sensor response of a sensing material ${ }^{(12)}$ is defined as

$$
S R=\left|R_{\mathrm{a}}-R\right| / R_{\mathrm{a}}
$$

where $R_{\mathrm{a}}$ is the resistance of the sensing element in air and $R$ is the resistance in a humid atmosphere.

\section{Results and Discussion}

Variations in the resistance with changes in \% $\mathrm{RH}$ for the sensing element of neodymium oxide annealed at different temperatures are shown in Fig. 5. It was observed that as \%RH inside the chamber increases from 15 to $95 \%$, the resistance of the sensing element decreases.

The curve in Fig. 5 for the sensing element prepared at $25^{\circ} \mathrm{C}$ has a lower gradient for the entire range of $\mathrm{RH}$ and has an average sensitivity of $7 \mathrm{M} \Omega / \% \mathrm{RH}$. The curve for the

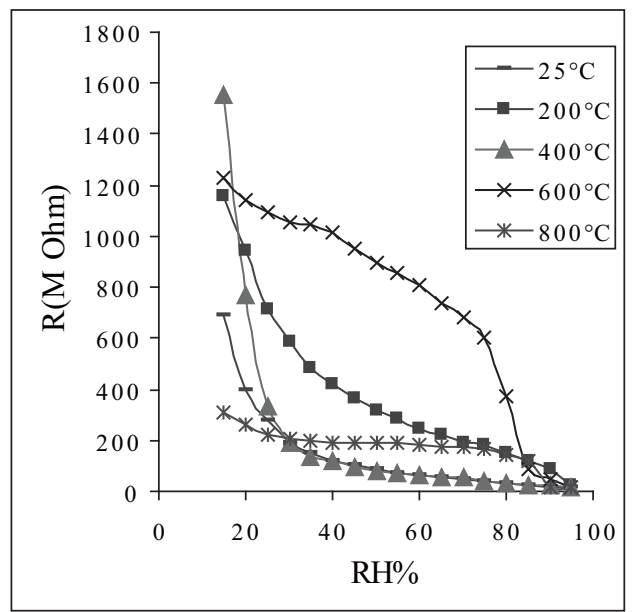

Fig. 5. Resistance vs \%RH for sensing element annealed at different temperatures. 
sensing element annealed at $200^{\circ} \mathrm{C}$ exhibits a steady decrease in resistance with increasing $\% \mathrm{RH}$ with higher sensitivity (14 M $/ \% \mathrm{RH})$. The curve for the sensing element annealed at $400{ }^{\circ} \mathrm{C}$ exhibits an abrupt decrease in resistance from 15 to $30 \% \mathrm{RH}$ with a maximum sensitivity of $92 \mathrm{M} \Omega / \% \mathrm{RH}$ and a slowly decreasing resistance at higher humidity. The average sensitivity is $19 \mathrm{M} \Omega / \% \mathrm{RH}$ over the entire range of $\mathrm{RH}$. The curve for the sensing element annealed at $600^{\circ} \mathrm{C}$ exhibits a different behaviour from the others. In the low humidity range, the change in resistance is slight, and in the higher humidity range, the resistance decreases rapidly resulting in high sensitivity. The average sensitivity is 15 $\mathrm{M} \Omega / \% \mathrm{RH}$. The curve for the sensing element annealed at $800^{\circ} \mathrm{C}$ shows the lowest slope over the entire range of RH and the lowest average sensitivity of $4 \mathrm{M} \Omega / \% \mathrm{RH}$.

The change in the impedance of porous ceramics at different humidities is related to the water adsorption mechanism on the oxide surface. The negatively charged oxygen of the water molecule is electrostatically attracted to the positively charged cationic side of the metal oxide surface. If the charge density of the cationic side is low, then water remains physically adsorbed at the surface by a weak electrostatic field. If the charge density of the cationic side is high, then water remains chemisorbed at the surface by a strong electrostatic field. The irreversible reaction at the first layer of water vapour adsorbed on metal oxide surface can be given as

$$
\mathrm{M}^{+}+\mathrm{H}_{2} \mathrm{O} \rightarrow \mathrm{M}-\mathrm{OH}+\mathrm{H}^{+} .
$$

Fripiat et al. and Anderson and Parks ${ }^{(13-14)}$ concluded that the weakening action of the surface electrostatic field promotes the dissociation of physisorbed water molecules in the following manner:

$$
2 \mathrm{H}_{2} \mathrm{O} \leftrightarrow \mathrm{H}_{3} \mathrm{O}^{+}+\mathrm{OH}^{-}
$$

The transport of charge carriers in pure water occurs by the attachment of a proton to a water molecule forming a hydronium ion. Without rotating, the hydronium ion donates another proton to a second water molecule, which accepts this proton, while a third proton becomes attached to a water molecule, and so on, throughout the liquid. This process is called a Grotthus chain reaction. The hydronium ion is most likely to be the charge carrier and responsible for the electrical conduction.

The sensitivities of the sensors prepared at different temperatures were also calculated. The sensitivity of the sensor prepared at room temperature was found to be $7 \mathrm{M} \Omega / \% \mathrm{RH}$, and the sensing element annealed at $400^{\circ} \mathrm{C}$ exhibited the highest average sensitivity (19 $\mathrm{M} \Omega / \% \mathrm{RH})$ over the entire range of $\mathrm{RH}$, i.e., from 15 to $95 \% \mathrm{RH}$. The variation of sensitivity with annealing temperatures of sensing element is shown in Fig. 6.

The reproducibility of results for sensors prepared at different temperatures was also studied. The best reproducibility was achieved at a higher range of RH for the sensing element annealed at $400^{\circ} \mathrm{C}$ as shown in Fig. 7. From the characteristics shown in Fig. 4, it is evident that the mass loss of the sensing material depends on the annealing temperature ${ }^{(15)}$ and a maximum mass loss of $11.43 \%$ is obtained as the temperature is increased from 200 to $400^{\circ} \mathrm{C}$. 


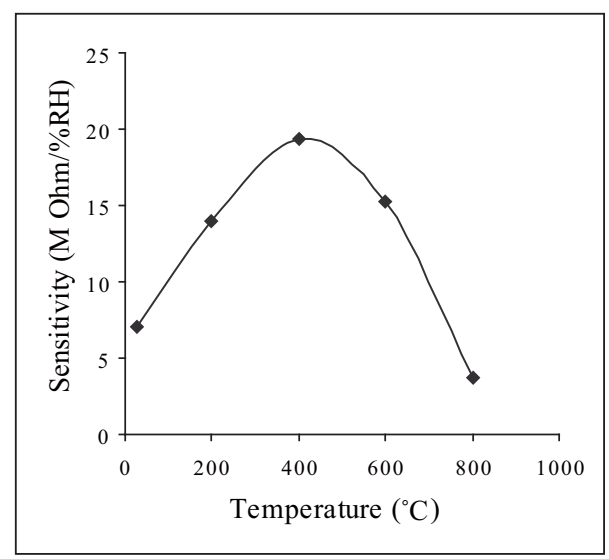

Fig. 6. Sensitivity vs annealing temperature of sensing element.

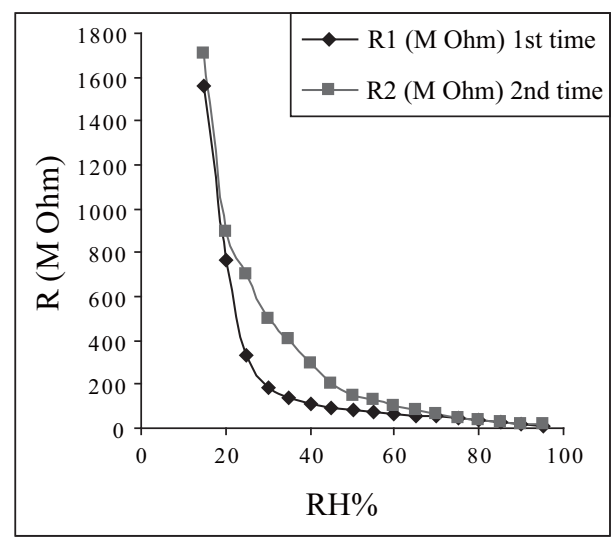

Fig. 7. Reproducibility of results for sensing element prepared at $400^{\circ} \mathrm{C}$.

\section{Conclusions}

The sensing element annealed at a temperature of $400^{\circ} \mathrm{C}$ was found to have better sensitivity than those annealed at other temperatures. Its average sensitivity was $19 \mathrm{M} \Omega$ / $\% \mathrm{RH}$ over a range of $\mathrm{RH}$ from 15 to $95 \%$. Results were found to be reproducible and no aging effect was observed. Thus, it was found that neodymium oxide nanoparticles exhibit good sensitivity to humidity. Our investigations are in the primary stage; however, we may conclude that this material will prove to be an excellent sensor for humidity measurements for future packaging and storages applications. 


\section{Acknowledgement}

The authors are thankful to Dr. C. D. Dwivedi for constant encouragement and discussions. We are also grateful to the Council of Science and Technology, Uttar Pradesh for providing financial support in the form of a project.

\section{References}

1 G. W. Brundrett: Criteria for Moisture Control (Butterworths, London, 1990) p. 12.

2 E. Traversa: Sens. Actuators, B 23 (1995) 135.

3 B. M. Kulwicki: J. Am. Ceram. Soc. 74 (1991) 697.

4 C. Y. Lee and G. B. Lee: Sens. Lett. 3 (2005) 1.

5 Z. Chen and C. Lu: Sens. Lett. 3 (2005) 274.

6 J. Singh, N. C. Soni and S. L. Srivastava: Bull. Mater. Sci. 4 (2003) 397.

7 A. A. Dakhel: Res.Technol. 39 (2004) 404.

8 W. Que, C. H. Kam, Y. Zhou, Y. L. Lam and Y. C. Chan: J. Appl. Phys. 90 (2001) 4865.

9 A. Biswas, I. G. Sharma, G. B. Kale and D. K. Bose: Metall. Mater. Trans. B 29 (1998) 309.

10 B. C. Yadav, R. Srivastava and C. D. Dwivedi: Synth. React. Inorg. Met.-Org. Nano-Met. Chem. 37 (2007) 1.

11 B. C. Yadav, R. Srivastava, C.D. Dwivedi and R. Kumar: Sens. Trans. 80 (2007) 1295.

12 N. Zhang, K. Yu, Z. Zhu and D. Jiang: Sens. Actuators, A 143 (2008) 245.

13 J. J. Fripiat, A. Jelli, G. Poncele and J. Andre: J. Phys. Chem. 69 (1965) 2185.

14 J. H. Anderson and G. A. Parks: J. Phys. Chem. 72 (1968) 3362.

15 J. Daun, X. Huang, E. Wang and H. Ai: Nanotechnology 17 (2006) 1786. 